\title{
Proporção sexual e comprimento de concha na primeira maturação sexual em Donax hanleyanus Philippi (Bivalvia, Donacidae) no Rio Grande do Sul, Brasil
}

\author{
Guacira Maria Gil \& José Willibaldo Thomé
}

Laboratório de Malacologia, Pontifícia Universidade Católica do Rio Grande do Sul. Avenida Ipiranga 6681, prédio 12-D, sala 340, Porto Alegre, 90619-900 Rio Grande do Sul.E-mail: guagil_8@hotmail.com; jwthome@pucrs.br

\begin{abstract}
Sex ratio and length shell at first maturity in Donax hanleyanus Philippi (Bivalvia, Donacidae) in Southern Brazil. Sex ratio and size at first maturity of the wedge clam Donax hanleyanus Philippi, 1847 were studied during 15 months, between October 2001 and December 2002, in Arroio Teixeira beach, southern Brazil. Circa of 3000 specimens were collected in swash zone and the length shell varied between 2.0 and $28.33 \mathrm{~mm}$. 347 specimens, between 7.0 and $28.3 \mathrm{~mm}$, were analyzed histologically. The sex ratio was 1:1. Different methods were employed in order to give a better idea of the mean size at first maturity. Donax hanleyanus attain maturity at mean lengths between $12.28 \mathrm{~mm}$ and $12.60 \mathrm{~mm}$. A minimum size of $15,0 \mathrm{~mm}$ (ca. eight months) for extraction is suggested.
\end{abstract}

KEY WORDS. Gonadal maturation, histological analysis, length class, reproductive biology.

RESUMO. Foi avaliada a proporção sexual e determinado o tamanho da concha, quando da primeira maturação sexual de uma população de Donax hanleyanus Philippi, 1847, amostrada durante 15 meses, entre outubro de 2001 e dezembro de 2002, na praia de Arroio Teixeira, sul do Brasil. Aproximadamente, 3000 espécimes foram coletados na zona de varrido, com comprimento de concha igual ou superior a 2,0 mm. A análise histológica foi realizada em 347 espécimes, entre 7,0 e 28,33 mm de comprimento de concha. A proporção sexual é de 1:1. Os diferentes métodos utilizados, para determinação do tamanho da primeira maturação sexual, resultaram em comprimentos aproximados, podendo-se concluir que Donax hanleyanus atinge a maturidade em um comprimento médio de concha entre 12,28 e $12,60 \mathrm{~mm}$. Sugere-se que o comprimento mínimo de captura seja estabelecido em $15,0 \mathrm{~mm}$.

PALAVRAS CHAVE. Análise histológica, biologia reprodutiva, classe de comprimento, maturação de gônada.

A costa do Rio Grande do Sul pertence à província faunística patagônica, caracterizada por abrigar espécies adaptadas a baixas temperaturas. O litoral gaúcho estende-se por $660 \mathrm{~km}$ de praias arenosas expostas, abrigando, dentre outras espécies, Donax hanleyanus Philippi, 1847, espécie filtradora que habita a zona intermareal rasa. A adaptação da espécie a essas praias de pequena declividade (GIAnuca 1983, Gil \& Thоме́ 2001a), com sedimento constituído por areia fina e quartzosa (MARTINs 1967, WesCHENFELDER et al. 1997), pode ser constatada pela freqüência e abundância que as populações de $D$. hanleyanus atingem, em todos os meses do ano, nas praias do Rio Grande do Sul (Gil \& Tноме́ 2001b).

Esta espécie tem sido objeto de pesquisas na Argentina, no Uruguai e no Brasil - os três países onde ocorre - e abordam, principalmente, aspectos anatômicos e ecológicos (PENCHASZADEH \& Olivier 1975, Narchi 1978, Defeo \& Alava 1995, Gil \& Thomé 2000, 2001a, Cardoso \& Veloso 2003).

Investigações sobre a reprodução e o desenvolvimento sexual da família Donacidae Fleming, 1828 (Bivalvia) têm sido realizadas por autores como MouËza \& Frenkiel-Renault (1973), Villiers (1975), Penchaszadeh \& Olivier (1975), Vélez (1985), Wegener (1996), Tirado \& Salas (1998, 1999), Zeichen et al. (2002), mas, no que tange à espécie $D$. hanleyanus, a despeito de sua abundância nas praias do Rio Grande do Sul e de sua importância ecológica no ecossistema de praias (GIL \& THомé 1998, Cardoso \& Veloso 2003), os processos reprodutivos da espécie ainda não foram bem compreendidos.

Neste estudo foi avaliada a proporção sexual e estimado o tamanho, quando da primeira maturação sexual, de uma população de D. hanleyanus, na praia arenosa de Arroio Teixeira, Rio Grande do Sul, Brasil.

\section{MATERIAL E MÉTODOS}

\section{Descrição da área}

A área escolhida para esta investigação foi a praia de Arroio Teixeira, que possui cerca de $6 \mathrm{~km}$ de extensão e está loca-

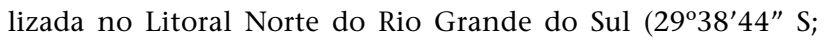

Revista Brasileira de Zoologia 21 (2): 345-350, junho 2004 
$\left.49^{\circ} 56^{\prime} 49^{\prime \prime} \mathrm{W}\right)$. Trata-se de uma praia arenosa exposta à arrebentação das ondas, apresentando estado morfodinâmico dissipativo (GIL \& THоме́ 2001a).

\section{Amostragem e procedimentos de laboratório}

As amostragens foram realizadas mensalmente, entre outubro de 2001 e dezembro de 2002, durante a maré baixa, utilizando-se um cilindro amostral de $25 \mathrm{~cm}$ de diâmetro e 10 $\mathrm{cm}$ de altura e uma peneira com malha de 2,0 $\mathrm{mm}$. Após a demarcação de três transectos perpendiculares à linha de praia, as coletas foram efetuadas na porção mediana da zona de varrido. Os exemplares foram fixados em formaldeído $4 \%$, com as valvas abertas, imediatamente após a coleta.

Para a avaliação da proporção sexual e determinação do comprimento de concha da primeira maturação sexual, foram selecionados apenas aqueles indivíduos cujo comprimento de concha fosse igual ou superior a 7,0 $\mathrm{mm}$, procedendo-se a análise histológica de gônadas de $50 \%$ dos indivíduos enquadrados neste critério.

O comprimento da concha $\left(\mathrm{L}_{\mathrm{t}}\right)$ foi medido com um paquímetro digital de $0,01 \mathrm{~mm}$ de precisão e, posteriormente, os animais foram agrupados por classes de comprimento de 1,0 mm (KREBS 1998).

A fim de determinar o sexo e verificar a condição de maturação sexual, procedeu-se a desidratação, diafanização e inclusão em parafina das gônadas de, aproximadamente, 20 indivíduos a cada mês. Posteriormente, estas foram seccionadas em cortes de $7 \mathrm{~mm}$ de espessura, coradas com hematoxilinaeosina, seguindo procedimento padrão (VéLez 1985) para análises histológicas. O comprimento da concha dos espécimes selecionados para os procedimentos histológicos variou de 7,0 $\mathrm{mm}$ a $28,33 \mathrm{~mm}$.

\section{Análise de dados}

Após uma análise preliminar dos cortes histológicos, foram distinguidos aqueles indivíduos indiferenciados sexualmente, daqueles que estavam em processo gametogênico. Para esses últimos, foram definidos quatro estágios de maturidade sexual, adaptando-se à espécie em estudo, as escalas propostas por NARCHI (1976) e Borzone et al. (2001).

A classificação dos indivíduos foi feita segundo a predominância de folículos em um dos estágios, pois, em um mesmo espécime, estes podem apresentar diferentes graus de maturação. Os estágios considerados apresentam as seguintes características:

Início da primeira maturação: presença de muitas células imaturas, de tamanho pequeno e uniforme, com material cromático fortemente corado. Espermatogônias e oogônias estão bem visíveis junto à parede dos folículos, que possuem diâmetro médio de $35 \mu \mathrm{m}$.

Repleção por gametas: estágio identificado, nos machos, pela presença de série espermática radial bem desenvolvida. Na maioria dos folículos gonadais, há o predomínio de espermátides e espermatozóides completamente maduros, ocupando a região central do lúmen. Nas fêmeas, os folículos estão expandidos, atingindo diâmetro médio de $200 \mu \mathrm{m}$. Os oócitos em vitelogênese apresentam forma elíptica, estando ligados à parede folicular por um pedúnculo.

Emissão/desova parcial com proliferação: fase em que ocorre a emissão/desova parcial de elementos sexuais maduros, mas os elementos proliferativos continuam sendo formados, indicando um ciclo contínuo de produção de gametas. Observam-se muitas gônias próximas à parede folicular. Muitos gametas ainda permanecem no lúmen e são liberados progressivamente, até o esvaziamento quase total do folículo.

Emissão/desova total: estágio caracterizado pela existência de folículos com paredes de formato irregular, contendo algumas células maduras residuais, no lúmen, e outras, células iniciais do processo reprodutivo, desprendidas da parede folicular, marcando o final da gametogênese. Em diferentes regiões das gônadas de um mesmo animal, pode-se constatar a reabsorção dos gametas residuais em alguns folículos e, em outros, o espessamento da parede, fato indicativo da retomada do ciclo reprodutivo.

A proporção sexual foi determinada a partir do número de fêmeas e machos obtidos nas amostras mensais e, para testar a hipótese de igualdade, foi aplicado o teste $\chi^{2}$ (ANSELl 1961, VAzZoler 1981, Vélez 1985).

Para a estimativa do comprimento médio da primeira maturação sexual $\left(\mathrm{L}_{\mathrm{m}}\right)$, foram testados dois dos métodos utilizados por Masello \& Defeo (1986): no primeiro, foi calculada a média (com intervalo de confiança em um nível de probabilidade de 95\%), levando-se em consideração o menor indivíduo no estágio de emissão/desova total, o menor indivíduo maduro e o maior indivíduo imaturo.

No segundo método foram construídos histogramas de freqüência de comprimento para indivíduos imaturos e para os demais indivíduos. O comprimento de concha, quando da primeira maturação sexual, foi definido como aquele a partir do qual a freqüência de maduros supera a de imaturos. Foram considerados imaturos o conjunto de indivíduos sexualmente indeterminados e aqueles em início de primeira maturação. Os demais foram denominados maduros.

\section{RESULTADOS E DISCUSSÃO}

Durante os 15 meses de amostragem, foram coletados 3103 animais com comprimento de concha variando entre $2,0 \mathrm{~mm}$ e $28,33 \mathrm{~mm}$. Desse total, 694 apresentaram comprimento da concha igual ou superior a 7,0 mm, tendo sido feita, então, a análise histológica das gônadas de 347 espécimes, número que corresponde a $50 \%$ do total de indivíduos.

Dentre a totalidade de espécimes analisados histologicamente, 27 (7,78 \%) eram animais indiferenciados, amostrados, principalmente, nos meses de abril, maio e junho, com comprimento de concha variando de $7,0 \mathrm{~mm}$ a $11,19 \mathrm{~mm} ; 43$ animais $(12,39 \%)$ encontravam-se em estágio inicial de proliferação das células sexuais (Figs 1 e 5), com comprimento de con-

Revista Brasileira de Zoologia 21 (2): 345-350, junho 2004 


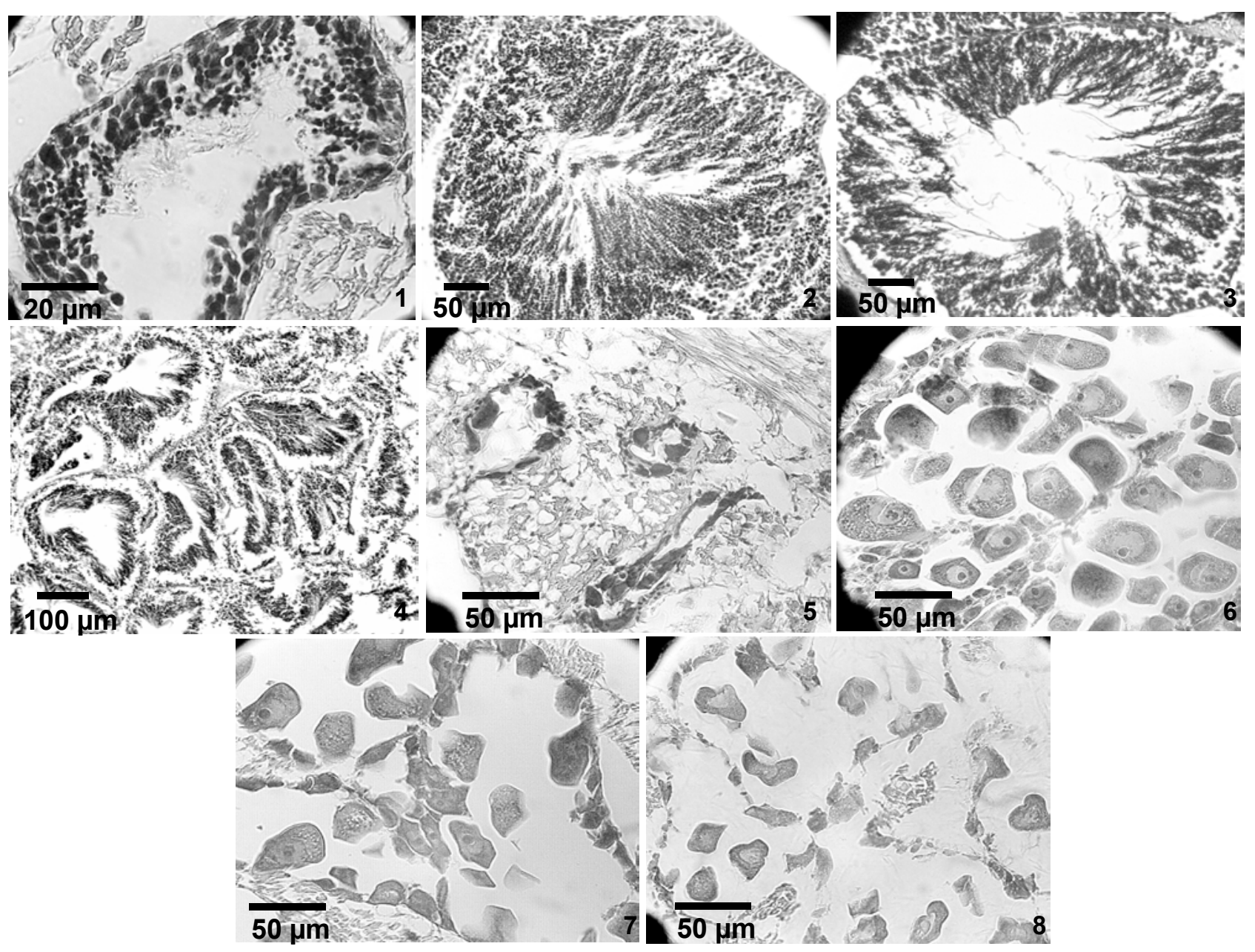

Figuras 1-8. Fotomicrografias de cortes histológicos: (1) macho em primeira maturação; (2) macho em repleção por gametas; (3) macho em emissão parcial; (4) macho em emissão total; (5) fêmea em primeira maturação; (6) fêmea em repleção por gametas; (7) fêmea em desova parcial; (8) fêmea em desova total.

cha entre 9,9 e 12,8 mm; 277 animais (79,83 \%) apresentavam gônadas em diferentes estágios de maturidade (Figs 2-4, 6-8), com comprimento de concha variando de 12,0 até $28,3 \mathrm{~mm}$.

Na tabela I são apresentados os comprimentos mínimo, máximo e médio de indivíduos adultos por estágio de maturação sexual e, também, daqueles sexualmente indeterminados. A identificação dos folículos gonadais, nesta população, tornouse possível em um comprimento médio de concha de 11,41 mm, nos machos, e $11,77 \mathrm{~mm}$, nas fêmeas, ainda que tenha sido encontrado um espécime macho em fase inicial de proliferação de células sexuais aos 9,90 mm de comprimento de concha.

Na população de $D$. hanleyanus, de Arroio Teixeira, o início do processo de amadurecimento gonadal ocorre em um comprimento de concha semelhante em machos e fêmeas. Já nas populações argentinas, é possível diferenciar os machos aos 4,0 $\mathrm{mm}$ (dois meses de idade) e, as fêmeas, aos 7,0 $\mathrm{mm}$ de comprimento médio de concha (quatro meses de idade) (Penchaszadeh \& Olivier 1975).

De modo semelhante ao que ocorre nas populações de D. trunculus Linnaeus, 1758, de Portugal (GASPAR et al. 1999), na população de $D$. hanleyanus, de Arroio Teixeira, machos e fêmeas apresentam uma sincronia nos estágios gametogênicos em relação aos meses do ano, indicando que a atividade reprodutiva está vinculada a fatores ambientais.

\section{Proporção sexual}

A análise histológica das gônadas confirma estudos prévios realizados por Penchaszadeh \& Olivier (1975) de que a espécie é gonocorística, não tendo sido encontrado nenhum indivíduo hermafrodita. Dos 347 espécimes analisados, 151 eram 
Tabela I. Comprimentos mínimos (Ltmin), máximos (Ltmax) e médios (Ltmed) de machos e fêmeas, por estágio reprodutivo e de indivíduos sexualmente indiferenciados.

\begin{tabular}{|c|c|c|c|c|c|c|}
\hline \multirow{2}{*}{ Estágio } & \multicolumn{3}{|c|}{ Fêmeas (mm) } & \multicolumn{3}{|c|}{$\operatorname{Machos}(\mathrm{mm})$} \\
\hline & Ltmin & Ltmax & Ltmed & Ltmin & Ltmax & Ltmed \\
\hline Início primeira maturação & 10,00 & 12,62 & 11,77 & 9,90 & 12,80 & 11,41 \\
\hline Repleção por gametas & 13,50 & 23,70 & 16,26 & 12,00 & 23,99 & 16,63 \\
\hline Emissão/Desova parcial & 12,00 & 28,33 & 17,29 & 12,00 & 22,73 & 16,98 \\
\hline Emissão/Desova total & 13,00 & 20,55 & 16,21 & 13,00 & 22,17 & 17,10 \\
\hline Indiferenciados (mm) & 7,00 & 11,19 & 9,86 & - & - & - \\
\hline
\end{tabular}

fêmeas, 169, machos e 27, sexualmente indiferenciados. Considerando-se apenas os indivíduos diferenciados, as fêmeas representaram 47,19 \% e, os machos, 52,81 \% da população amostrada. Esta pequena preponderância de machos parece ser comum nas espécies do gênero (ANSELl 1983, GaSPar et al. 1999).

O número de indivíduos por sexo, o comprimento médio e respectivo desvio padrão para machos e fêmeas, por mês de amostragem e o teste $\chi^{2}$ para testar a proporção entre os sexos, a cada mês, são apresentados na tabela II. A hipótese de que o número de machos e fêmeas na população tende à igualdade (1:1) foi confirmada, após ter sido aplicado o teste $\chi^{2}(\alpha=0,05)$, para o total de amostras, utilizando-se o pacote estatístico Statistica for Windows, versão 5.1, $\left(\chi^{2}=4,05 ; \mathrm{df}=14 ; \mathrm{p}<0,9975\right)$. O resultado obtido confirma as afirmativas de CoE (1953) sobre a distribuição igualitária de machos e fêmeas na natureza, e de Ansell (1983), de que no gênero Donax Linnaeus, 1758, a proporção sexual é de 1:1, aproximadamente.

\section{Primeira maturação sexual}

Os dois métodos utilizados, para avaliar o comprimento médio da primeira maturação sexual, resultaram em valores próximos: $12,60 \mathrm{~mm}$ e $12,28 \mathrm{~mm}$.

No primeiro método, no qual se calculou a média entre os comprimentos do menor indivíduo no estágio de emissão/ desova total $(13,0 \mathrm{~mm})$, do menor indivíduo maduro $(12,0 \mathrm{~mm})$ e do maior indivíduo imaturo $(12,80 \mathrm{~mm})$, o valor resultante foi de $12,60 \mathrm{~mm}$.

O segundo método, que considera a distribuição de freqüência de exemplares imaturos e de maduros para determinação do comprimento da primeira maturação sexual (Fig. 9), resultou em um valor de $12,28 \mathrm{~mm}$.

Os dois métodos utilizados levam-nos a concluir que, nesta população, o desenvolvimento da gônada inicia-se em um $\mathrm{L}_{\mathrm{t}}$ médio de $11,60 \mathrm{~mm}$, quando estão em uma idade aproximada de cinco meses (GIL \& Thomé 2000). A primeira matu-

Tabela II. Número de machos e fêmeas por mês de amostragem, teste $\chi^{2}$, e comprimentos médios (Ltmed) e desvio padrão (dp).

\begin{tabular}{|c|c|c|c|c|c|}
\hline \multirow{2}{*}{ Mês } & \multirow{2}{*}{ Machos } & \multirow{2}{*}{ Fêmeas } & \multirow{2}{*}{$\chi^{2}$} & Ltmed ( dp (mm) & \multirow{2}{*}{$\frac{\text { Ltmed }(\mathrm{dp}(\mathrm{mm})}{\text { Fêmeas }}$} \\
\hline & & & & Machos & \\
\hline Out/2001 & 14 & 16 & 0,133 & $13,82-1,23$ & $13,33-0,97$ \\
\hline Nov/2001 & 14 & 14 & 0,000 & $15,12-1,88$ & $15,35-1,31$ \\
\hline Dez/2001 & 9 & 13 & 0,727 & $14,02-2,86$ & $14,38-2,87$ \\
\hline Jan/2002 & 13 & 12 & 0,040 & $15,42-2,51$ & $14,42-1,81$ \\
\hline Fev/2002 & 10 & 10 & 0,000 & $16,58-3,37$ & $16,94-2,37$ \\
\hline Mar/2002 & 13 & 11 & 0,166 & $17,25-3,26$ & $18,05-4,85$ \\
\hline Abri/2002 & 9 & 9 & 0,000 & $18,09-3,70$ & $17,96-4,38$ \\
\hline Maio/2002 & 10 & 9 & 0,052 & $16,02-4,85$ & $16,11-4,41$ \\
\hline Jun/2002 & 9 & 6 & 0,600 & $14,60-5,86$ & $18,39-4,82$ \\
\hline Jul/2002 & 14 & 6 & 3,200 & $14,88-1,38$ & $14,49-1,49$ \\
\hline Ago/2002 & 13 & 6 & 2,578 & $15,88-3,22$ & $15,00-2,59$ \\
\hline Set/2002 & 11 & 9 & 0,200 & $15,82-3,47$ & $16,11-3,61$ \\
\hline Out/2002 & 9 & 11 & 0,200 & $15,67-2,46$ & $16,55-2,66$ \\
\hline Nov/2002 & 11 & 9 & 0,200 & $13,86-2,50$ & $14,28-2,24$ \\
\hline Dez/2002 & 10 & 10 & 0,000 & $17,75-1,40$ & $16,00-1,41$ \\
\hline
\end{tabular}

Revista Brasileira de Zoologia 21 (2): 345-350, junho 2004 


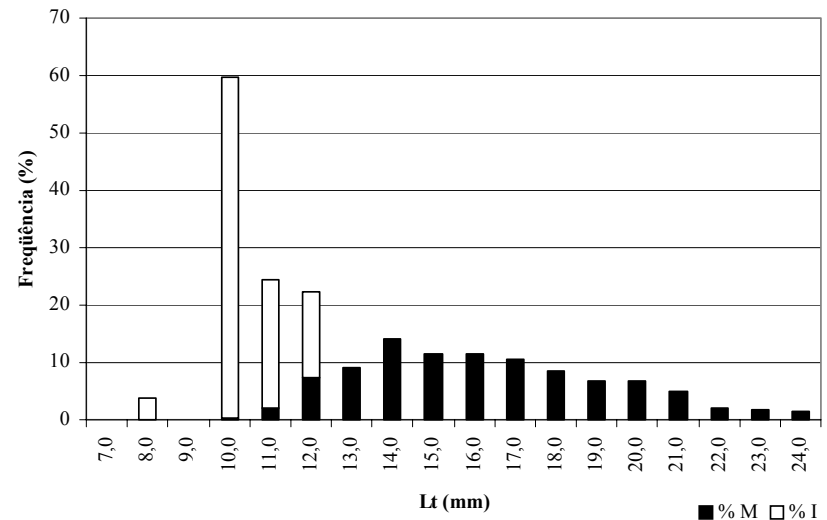

Figura 9. Distribuição de freqüência (\%) de indivíduos imaturos (I) e maduros $(M)$, por classe de comprimento $\left(L_{t}\right)$.

ração sexual ocorre quando a maioria dos indivíduos está entre 12,0 e $12,80 \mathrm{~mm}$ de comprimento médio de concha (entre cinco e seis meses de idade) e, a partir deste tamanho, $100 \%$ dos indivíduos apresentam gônadas diferenciadas.

Em estudo prévio, GiL \& Tноме́ (2000) constataram que há dois picos reprodutivos anuais na população de D. hanleyanus. Porém, a existência de jovens em praticamente todos os meses do ano, forneceu um indicativo de que o processo reprodutivo, nesta população, é contínuo. No presente trabalho, o fato de terem sido encontrados animais em todos os estágios reprodutivos, em qualquer época do ano, reforça este indicativo, confirmando que, após o amadurecimento sexual, D. hanleyanus passa a se reproduzir, potencialmente, em todos os meses.

Os resultados aqui apresentados revelam que os animais com comprimento de concha inferior a $12,0 \mathrm{~mm}$ ainda não atingiram a maturidade sexual. Também se pode constatar que a maior freqüência de indivíduos em atividade sexual encontra-se entre as classes de comprimento de 13,0 a 15,0 $\mathrm{mm}$.

O fato de suas populações viverem em uma zona de fácil acesso (varrido), faz com que esses animais terminem por ser alvo de coletas indiscriminadas por parte de veranistas e moradores do litoral, que os utilizam para consumo próprio, na alimentação e como iscas para pesca ou, ainda, os comercializam em pequenas peixarias.

Considerando-se as condições acima descritas e os resultados obtidos, sugerimos que o comprimento mínimo de captura de $D$. hanleyanus seja estabelecido em 15,0 mm, comprimento em que a maioria dos indivíduos da população já se reproduziu, pelo menos, uma vez. Sugerimos, também, que haja um acompanhamento sistemático dessas populações, por parte dos órgãos ambientais, como forma de controlar e de manter os estoques.

\section{AGRADECIMENTOS}

Agradecemos o apoio financeiro do CNPq e FAPERGS e o auxílio da bolsista de Iniciação Científica Fernanda Toscani e da estagiária Carolina Heinz, pelo processamento do material histológico.

\section{REFERÊNCIAS BIBLIOGRÁFICAS}

ANSELL, A.D. 1961. Reproduction, growth and mortality of Venus striatula (Da Costa) in Kames Bay, Millport. Journal of Marine Biology Association UK, London, 41: 191-215.

. 1983. The biology of genus Donax, p. 607-635. In: A. Mclachlan \& T. Erasmus (Eds). Sandy beach as ecosystems. The Hague, W. Junk, 756p.

Borzone, C.A.; K.M. Vargas; P.R. Pezzuto \& Y.A.G. Tavares. 2001. Aspectos da reprodução e dinâmica populacional de Chione pubera (Bory Saint-Vicent) (Bivalvia: Veneridae) no sul do Brasil. Revista Brasileira de Zoologia, Curitiba, 18 (2): 333-349.

Cardoso, R.S. \& V.G. Veloso. 2003. Population dynamics and secondary production of the wedge clam Donax hanleyanus (Bivalvia: Donacidae) on a high-energy, subtropical beach of Brazil. Marine Biology, Berlin, 142: 153-162.

CoE, W.R. 1953. Resurgent populations of littoral marine invertebrates and their dependence on ocean currents and tidal currents. Ecology, La Jolla, 34: 225-229.

Defeo, O. \& A. Alava. 1995. Effects of human activities on longterm trends in sandy beach populations: the wedge clam Donax hanleyanus in Uruguay. Marine Ecological Progress Serie, Ordendorf/Luhe, 123: 73-82.

Gaspar, M.B.; R. Ferreira \& C.C. Monteiro. 1999. Growth and reproductive cycle of Donax trunculus L. (Mollusca: Bivalvia) off Faro, southern Portugal. Fisheries Research, New York, 41: 309-316.

GianUCA, N.M. 1983. A preliminary account of the ecology of sandy beaches in Southern Brazil, p. 413-419. In: A. McLAchlan \& T. ERasmus (Ed.). Sandy beach as ecosystems. The Hague, W. Junk, 756p.

G.M. GIL \& J.W. Thomé. 1998. Donax hanleyanus Philippi, 1847 como indicador ambiental. Biociências, Porto Alegre, 6 (2): 189-193.

- 2000. Estudo do crescimento em comprimento de Donax hanleyanus Philippi, 1847 (Mollusca, Bivalvia, Donacidae). Biociências, Porto Alegre, 8 (2): 163-175.

. 2001a. Influência de variáveis ambientais sobre uma população de Donax hanleyanus Philippi, 1847 (Mollusca, Bivalvia, Donacidae). Biotemas, Florianópolis, 14: 109-126.

. 2001b. Abundância, freqüência e densidade relativa da malacofauna da praia de Arroio Teixeira, Rio Grande do Sul. Revista Brasileira de Zoociências, Juiz de Fora, 14: 127-136.

KreBS, C.J. 1998. Ecological Methodology. Menlo Park, Addison Wesley Longman, 620p.

Martins, L.R. 1967. Aspectos texturais e deposicionais dos sedimentos praiais e eólicos da planície costeira do Rio Grande do Sul. Publicação Especial da Escola de Geologia da UFRGS, Porto Alegre, 13: 1-102.

Masello, A. \& O. Defeo. 1986. Determinación de la longitud de primera madurez sexual en Mesodesma mactroides (Deshayes, 1854). Comunicaciones de la Sociedad Malacologica del

Revista Brasileira de Zoologia 21 (2): 345-350, junho 2004 
Uruguay, Montevideo, 6: 387-395.

MouËZa, M. \& L. Frenkiel-Renault. 1973. Contribution à l'étude de la biologie de Donax trunculus L. (Mollusques Lamellibranches) dans l'Algèrois: la reproduction. Cahiers Biologie Marine, Paris, 14: 261-283.

NARCHI, W. 1976. Ciclo anual da gametogênese de Anomalocardia brasiliana (Gmelin, 1791) (Mollusca Bivalvia). Boletim de Zoologia da Universidade de São Paulo, São Paulo, 1: 331350 .

. 1978. Functional anatomy of Donax hanleyanus Philippi, 1847 (Donacidae: Bivalvia). Boletim de Zoologia da Universidade de São Paulo, São Paulo, 3: 121-142.

Penchaszadeh, P.E. \& S.R. Olivier. 1975. Ecología de una población de "berberecho" (Donax hanleyanus) en Villa Gesell, Argentina. Malacologia, Ann Arbor, 15 (1): 133-146.

Tirado, C. \& C. Salas. 1998. Reproduction and fecundity of Donax trunculus L. 1758 (Bivalvia: Donacidae) in the littoral of Malaga (Southern Spain). Journal of Shellfishery Research, Miami, 17: 169-176.

- 1999. Reproduction of Donax venustus Poli, 1795, Donax semistriatus Poli, 1795 and intermediate morphotypes (Bivalvia: Donacidae) in the littoral of Málaga (Southern
Spain). Marine Ecology, Nápoli, 20: 111-130.

VAzzoler, A.E.A.M. 1981. Manual de métodos para estudos biológicos de populações de peixes: reprodução e crescimento. Brasília, CNPq, 106p.

VéLez, A.V. 1985. Reproductive biology of the tropical clam Donax denticulatus in eastern Venezuela. Caribbean Journal of Science, Mayagüez, 21 (3-4): 125-136.

VILLIERS, G. DE. 1975. Reproduction of the sand mussel Donax serra Röding, Investigation on the Sea Fisheries of the Republic South Africa, Pretoria, 102: 1-33.

Wegener, A. 1996. Determination of reproductive cycle in a population of Egeria radiata (Lamarck, 1804) (Bivalvia: Donacidae) using condition index and histological examination of gonads. Annales de Limnologie, Paris, 32: 105-113.

Weschenfelder, J.; R.N.A. Zouain; S.L.C. Zomer \& R.P. Souto. 1997. Caracterização morfológica das praias oceânicas entre Imbé e Arroio do Sal, RS. Notas Técnicas, Porto Alegre, 10: 35-48.

Zeichen, M.M; S. Agnesi; A. Mariani; A. Maccaroni \& G.D. Ardizzone. 2002. Biology and population dynamics of Donax trunculus L. (Bivalvia: Donacidae) in South Adriatic Coast. Estuarine, Coastal and Shelf Science, London, 54 (6): 971-982.

Recebido em 11.XI.2003; aceito em 05.VI.2004.

Revista Brasileira de Zoologia 21 (2): 345-350, junho 2004 